\title{
A Provably Stable and Simple FDTD Formulation for Electromagnetic Modeling of Graphene Sheets
}

\author{
Fatemeh Afshar, Ali Akbarzadeh-Sharbaf, and Dennis D. Giannacopoulos
}

Department of Electrical and Computer Engineering, McGill University, Montreal QC H3A 0E9, Canada

\begin{abstract}
A new finite-difference time-domain (FDTD) formulation for modeling graphene is proposed, in which the graphene is modeled as a resistive sheet with a frequency-dependent conductivity. The formulation is first developed in the context of the vector wave finite-element time-domain and then reduced to the FDTD based on the equivalence between these two techniques. The obtained formulation is easy to implement and does not alter the original FDTD update equations. It can be applied to an existing FDTD code by simply adding a correction term to appropriate variables. One of the main contributions of this paper is analyzing the stability of the proposed formulation, which has not been done previously.
\end{abstract}

Index Terms-Boundary conditions, finite-difference methods, finite-element analysis, graphene.

\section{INTRODUCTION}

I NVENTED in 2004, graphene is often called a revolutionary material of the 21 st century. Due to its outstanding electrical, mechanical, magnetic, and thermal properties, graphene has recently gained significant interest among scientists. Its conductivity can be tuned to be metal- or semiconductorlike, which makes it an excellent candidate for high-frequency electronics [1]. Many graphene-based applications, such as waveguides, nanoantennas, or electromagnetic (EM)-shielding, have been introduced in recent years; thus, the need for studying their EM behavior has emerged. Since obtaining the analytic solution of Maxwell's equations in most cases is impossible, the use of numerical simulation methods is helpful. Graphene has been modeled using different methods, such as method of moments [2], finite-element method [3], and finitedifference time-domain (FDTD) method [4]-[8].

In a macroscopic EM fields context, the graphene acts like a thin surface with conductivity that depends on chemical doping or external fields [9]. Up to now, three approaches have been used to model the graphene in FDTD methods which are: 1) regular FDTD method with very fine field discretization in the graphene layer [4], [5]; 2) the sub-cell FDTD approach [6]; and 3) the graphene as a surface boundary condition in FDTD [7]. Since the graphene is very thin (one atomic thick layer), the first approach will be very expensive requiring a significant amount of memory and time, which makes it a poor option in practice. In spite of the merits of the other two approaches, they share at least two major drawbacks: first, they require different update equations in the vicinity of the graphene sheet, which makes the programming difficult; and second, the effect of the modified equations on the stability of the underlying FDTD has not been analytically studied.

In this paper, the graphene is considered as a resistive sheet, as in [7]; however, the formulation is inspired by the

Manuscript received July 4, 2015; revised September 3, 2015; accepted September 30, 2015. Date of publication October 7, 2015; date of current version February 17, 2016. Corresponding author: F. Afshar (e-mail: fatemeh.afshar@mail.mcgill.ca).

Color versions of one or more of the figures in this paper are available online at http://ieeexplore.iee.org.

Digital Object Identifier 10.1109/TMAG.2015.2487835 finite-element time-domain (FETD) method based on the vector wave equation (VWE) [8]. The formulation is then reduced to the FDTD method by evaluating the FETD integrals using the trapezoidal rule. The proposed FDTD formulation can be implemented with minimal modification on an existing FDTD code. In addition, because of the equivalence between FDTD and FETD, the stability of the proposed FDTD method can be analyzed by finding the location of the eigenvalues in the FETD formulation.

\section{FORMULATION}

\section{A. Graphene Conductivity Model}

Graphene has a frequency-dependent complex-valued conductivity. The macroscopic graphene conductivity model used in the FDTD method consists of two terms: 1) interband conductivity and 2) intraband conductivity. The graphene surface conductivity (in units of $[\mathrm{S}]$ ) is given by the Kubo formula in an integral form. The simplified Kubo formula for the intraband conductivity term is [10]

$$
\sigma_{\text {intra }}\left(\omega, \mu_{c}, \gamma, T\right)=\frac{-j \alpha}{\omega-j 2 \gamma}
$$

in which $\alpha=\left(e^{2} k_{B} T / \pi \hbar^{2}\right)\left(\left(\mu_{c} / k_{B} T\right)+2 \ln \left(e^{\left(-\mu_{c} / k_{B} T\right)}+1\right)\right)$, $\omega$ is the angular frequency in $\mathrm{rad} / \mathrm{s}$, and $\gamma$ is the scattering rate in $\mathrm{s}^{-1}$. In addition, $\mu_{c}$ is the chemical potential in $\mathrm{eV}$, which can be controlled by chemical doping or by applying a bias voltage, $T$ is the temperature in Kelvin, $e$ is the electron charge, $\hbar$ is the reduced Planck's constant, and $k_{B}$ is the Boltzmann constant. The reason why only the intraband conductivity has been considered here is because in microwave frequencies, in which we are interested here, the interband term can be neglected. As seen in (1), the intraband contribution is expressed by a simple Drude-like expression [11] and can be directly implemented in FDTD formulation, whereas the interband term cannot.

\section{B. Finite-Element Formulations in Time-Domain}

By expanding electric field and magnetic field variables in terms of edge (Whitney 1-form) and face (Whitney 2-form) elements, we will have two main FETD formulations, one of 


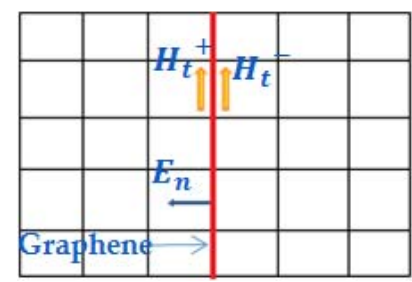

Fig. 1. Graphene sheet in a 2-D rectangular grid.

which is based on the VWE

$$
[T] \frac{d^{2}\{e\}}{d t^{2}}+[S]\{e\}=\{f\}
$$

while the second one involves directly solving two coupled Maxwell's equations

$$
\left\{\begin{array}{l}
{[T] \frac{d\{e\}}{d t}=[C]^{T}\left[T_{f}\right]\{b\}} \\
\frac{d\{b\}}{d t}=-[C]\{e\}
\end{array}\right.
$$

where $\{e\}=\left[e_{1}, e_{2}, \ldots, e_{N}\right]^{T}$ and $\{b\}=\left[b_{1}, b_{2}, \ldots, b_{N_{f}}\right]^{T}$ denote the electric field intensity and magnetic flux density unknowns, respectively. $[C]$ represents the incident matrix, which is sparse and has $-1,0$, or +1 entries, and $[T],\left[T_{f}\right]$, and $[S]$ are the square matrices given by

$$
\begin{aligned}
T_{i j} & =\int \epsilon W_{i}^{(1)} \cdot W_{j}^{(1)} d \Omega \\
S_{i j} & =\int \mu^{-1} \nabla \times W_{i}^{1} \cdot \nabla \times W_{j}^{(1)} d \Omega \\
T_{f, i j} & =\int \mu^{-1} W_{i}^{(2)} \cdot W_{j}^{(2)} d \Omega
\end{aligned}
$$

in which $W^{(1)}$ and $W^{(2)}$ represent the Whitney 1-form and Whitney 2-form elements, respectively.

By eliminating $\{b\}$ in (3), we obtain the VWE formulation (2) in which it can be shown that $[S]=[C]^{T}\left[T_{f}\right][C]$ for Whitney elements [12]. Therefore, both formulations become equivalent to each other in the continuous-time case. If the $[T]$ and $\left[T_{f}\right]$ matrices, and $[S]$ in $3-\mathrm{D}$ case, are approximated using the trapezoidal integration rule, it can be shown that (3) can exactly lead to the standard FDTD equations in space [13].

\section{Modeling of Graphene in FETD}

Fig. 1 shows a 2-D rectangular grid in which the electric field unknowns $\{e\}$ are assumed to be represented by edges. Graphene can be considered as a resistive sheet on which we have

$$
\sigma_{\text {intra }} E=\hat{n} \times\left(H^{+}-H^{-}\right) .
$$

The resistive sheet can be modeled in the VWE-FETD simply [8] as

$$
[T] \frac{d^{2}\{e\}}{d t^{2}}+[S]\{e\}+\{g\}=\{0\}
$$

in which

$$
\{g\}=\frac{\sigma \mathfrak{D}}{1+\tau \mathfrak{D}}\left[Q_{g}\right]\{e\}
$$

where $\mathfrak{D}$ represents $\partial / \partial t$ which replaces $j \omega$ in time-domain, $\sigma=(\alpha / 2 \gamma)$, and relaxation time $\tau=(1 / 2 \gamma)$ and

$$
Q_{g_{i j}}=\int_{\text {Graphene }} \hat{n} \times W_{i}^{(1)} \cdot \hat{n} \times W_{j}^{(1)} d s .
$$

Equation (8) can be transformed into the mixed FETD formulation in which both electric $\{e\}$ and magnetic fields $\{b\}$ are being updated together similar to the FDTD as

$$
\left\{\begin{array}{l}
{[T] \frac{\{e\}^{n+1}-\{e\}^{n}}{\Delta t}=[C]^{T}\left[T_{f}\right]\{b\}^{n+\frac{1}{2}}-\{J\}^{n+\frac{1}{2}}} \\
\frac{\{b\}^{n+\frac{3}{2}}-\{b\}^{n+\frac{1}{2}}}{\Delta t}=-[C]\{e\}^{n+1}
\end{array}\right.
$$

where $\partial J / \partial t=\{g\}$. Applying the central difference scheme to this term yields

$$
\{J\}^{n+\frac{1}{2}}=\{J\}^{n-\frac{1}{2}}+\Delta t\{g\}^{n} .
$$

In order to obtain $g^{n}$, we discretize (9) with the trapezoidal rule by simply replacing $\mathfrak{D}$ with the following bilinear transformation [14]:

$$
\frac{2}{\Delta t} \frac{1-z^{-1}}{1+z^{-1}}
$$

and invoking the $z$-transform properties, which gives

$$
\{g\}^{n}=\left(\frac{2 \tau-\Delta t}{2 \tau+\Delta t}\right)\{g\}^{n-1}+\frac{2 \sigma\left[Q_{g}\right]\left(\{e\}^{n}-\{e\}^{n-1}\right)}{(2 \tau+\Delta t)} .
$$

In this paper, we have employed the central difference to discretize the mixed FETD (11) to exactly recover the leapfrog FDTD out of it, although applying other schemes, such as Crank-Nicolson or alternating direction implicit, is readily possible. The trapezoidal integration has to be employed to evaluate the integrals, which makes $[T],\left[T_{f}\right]$, and $\left[Q_{g}\right]$ fully diagonal (mass lumping procedure). It should be noted that only those unknowns which lie on the graphene sheet have non-zero contribution in $\left[Q_{g}\right]$. Having substituted masslumped matrices in (11), update equations identical to the fully discretized FDTD are obtained in which the electric field update equation for those unknown resided on the graphene sheet has an additional term $\{\boldsymbol{J}\}$. In case of an explicit FDTD method, e.g., the standard leapfrog FDTD discussed here, $\{\boldsymbol{J}\}$ can be updated separately and applied as a correction term after the standard update process is performed, which greatly simplifies implementation. Evidently, the update equation for the magnetic field is not changed in this approach.

\section{Stability Analysis}

Since our method is based on the equivalence between FETD and FDTD method, they have the same stability criteria. Therefore, for the sake of simplicity, we study the stability analysis of VWE-FETD.

In order to simplify the stability analysis, we have considered a rectangular grid with the graphene on every edge of the grid. A perfect electric conductor (PEC) boundary condition is used to truncate the computational domain. Since in our grid $\Delta x=\Delta y$, both $[T]$ and $\left[Q_{g}\right]$ will be diagonal matrices each 
with the same entry on the diagonal. By substituting $[T]=\beta I$ and $\left[Q_{g}\right]=\varphi I$, we will have

$$
\beta I \frac{d^{2}\{e\}}{d t^{2}}+[S]\{e\}+\varphi I \frac{\sigma \mathfrak{D}}{1+\tau \mathfrak{D}}\{e\}=0 .
$$

For a square grid, $\beta=\epsilon \Delta x^{2}$ and $\varphi=\Delta x$. Since $[S]$ is a symmetric matrix, it can be written as $[S]=[P]^{-1}[V][P]$, by multiplying (15) is $[P]^{-1}$ and replacing $\tilde{e}=[P]^{-1} e$, our equations will be decoupled and with $\lambda_{i}$ as the eigenvalues of $[S]$, we will have

$$
\beta \frac{d^{2}\{\tilde{e}\}}{d t^{2}}+\lambda_{i}\{\tilde{e}\}+\varphi \frac{\sigma \mathfrak{D}}{1+\tau \mathfrak{D}}\{\tilde{e}\}=0 .
$$

Discretizing $\tilde{e}$ by central difference and the conductivity term via the trapezoidal method, we will obtain a third-order polynomial. By investigating the roots of the characteristic polynomial using Routh-Hurwitz stability criterion [15], and to have all eigenvalues of the amplification matrix reside inside or on the unit circle, we reach this condition

$$
\Delta t \leq \frac{2}{\sqrt{\varphi \sigma \beta^{-1} \tau^{-1}+\beta^{-1} \lambda}} .
$$

According to the definition of $\lambda$ and $\beta, \beta^{-1} \lambda$ is representing the eigenvalues of $[T]^{-1}[S]$. The most limiting $\Delta t$ is corresponding to the maximum value of $\beta^{-1} \lambda$, which is shown to be equal to

$$
\beta^{-1} \lambda=\frac{4 c^{2}}{\Delta x^{2}}+\frac{4 c^{2}}{\Delta y^{2}}
$$

for 2-D case [16], in which $c$ is the speed of light. As can be seen from (17), this condition reduces to the CFL condition in the absence of graphene $(\sigma=0)$. However, it becomes more limiting, if the graphene layers are included in simulation. In order to see how much more stringent the new condition is, we consider the ratio of the two terms in the denominator of (17). By substituting right-hand side of (18), $\beta=\epsilon \Delta x^{2}$ and $\varphi=\Delta x$, we have

$$
\frac{\varphi \sigma \beta^{-1} \tau^{-1}}{\lambda \beta^{-1}}=\frac{\mu \sigma \Delta x}{8 \tau}
$$

which is very small for typical graphene problems. In order to demonstrate the validity of the stability condition, we conducted a numerical example in which we considered a rectangular grid with $30 \times 30$ cells, $\Delta x=\Delta y=2 \mu \mathrm{m}$, and PEC boundary condition on the outer boundaries. We assumed that the graphene resides on every edge of the grid with $T=300 \mathrm{~K}, \mu_{c}=0.5 \mathrm{ev}$, and $\tau=0.5 \mathrm{ps}$. Under this assumptions, the CFL condition yields $4.717 \times 10^{-15}$, and the condition obtained from (17) gives $4.632 \times 10^{-15}$, which is $<2 \%$ smaller than the CFL condition. We performed the simulation for two different values of $\Delta t$ slightly below and above the value obtained from the new condition, which are $4.63 \times 10^{-15}$ and $4.64 \times 10^{-15}$, respectively. As can be seen in Fig. 2, the first $\Delta t$ value results in completely stable results, while the second value of $\Delta t$ yields to unstable results.

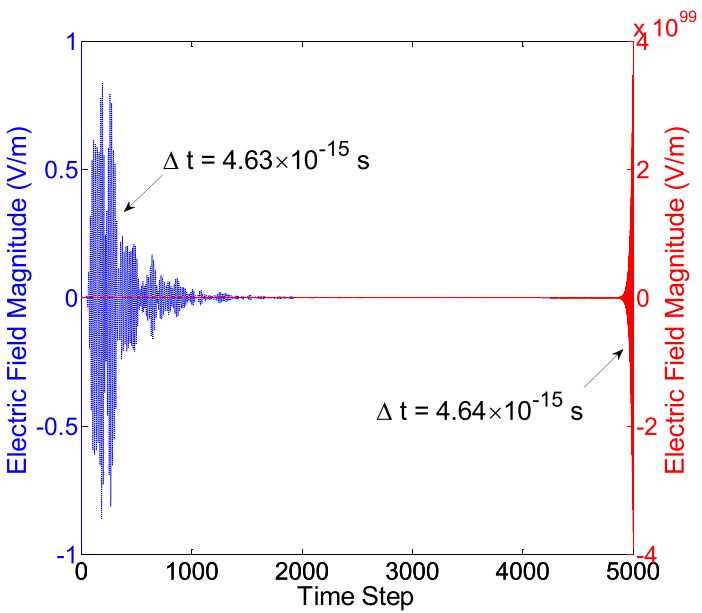

Fig. 2. Stability analysis. Left: stable results with time steps slightly below the new stability condition. Right: unstable results with time steps slightly above the new stability condition.

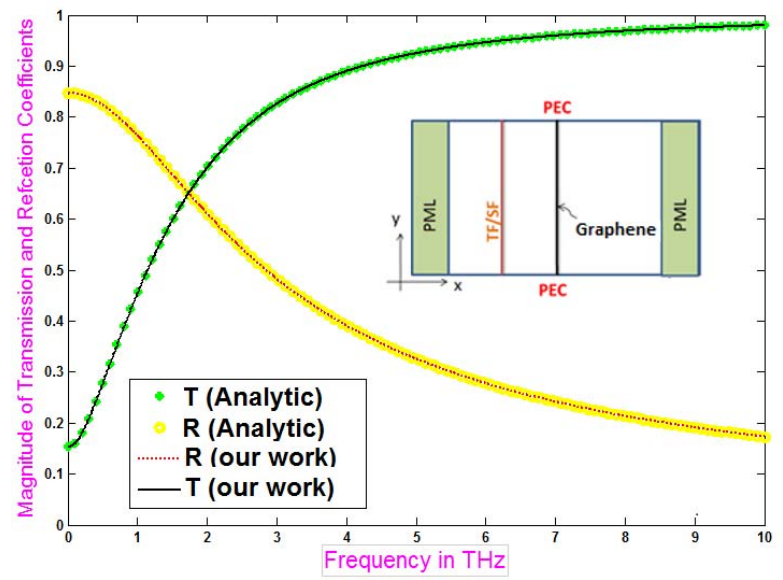

Fig. 3. Comparison between transmission and reflection coefficients of our numerical method and analytical results for graphene. $T=300 \mathrm{~K} . \tau=0.5$ ps. $\mu c=0.5 \mathrm{ev}$.

\section{Numerical Results}

In order to validate the proposed formulation, we considered two numerical examples. Our first example involves calculating reflection and transmission coefficients of an infinitely long graphene sheet with $T=300 \mathrm{~K}, \mu_{c}=0.5 \mathrm{ev}$, and $\tau=0.5 \mathrm{ps}$ as in [10], located in the middle of our computational domain. The problem was solved in 2-D with $210 \times 210$ cells, $\Delta x=\Delta y=2 \mu \mathrm{m}$. A PEC was used to truncate the graphene layer and an eight-cell perfectly matched layer (PML) for both sides of the domain. We excited the problem with a Blackman-Harris pulse-shaped plane wave [11]. The exact solutions were calculated by $T=2 /\left(2+\eta_{0} \sigma_{\mathrm{gr}}\right)$ and $\Gamma=T-1$, in which $\eta_{0}$ is the free-space impedance and $\sigma_{\mathrm{gr}}$ is the graphene conductivity.

Fig. 3 shows the comparison of the numerical and analytical results of transmission and reflection coefficients, in which we can observe that our method is in excellent agreement with the analytical results.

Graphene has unique properties and can support surface plasmon polaritons (SPPs). The field of SPPs supported by the graphene is tightly confined on the surface, and the damping 


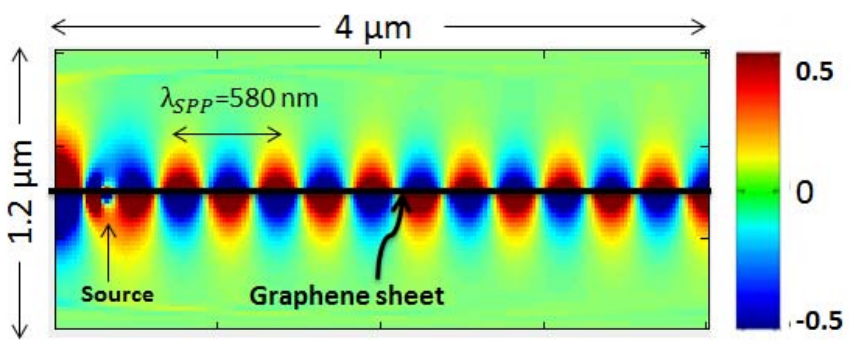

Fig. 4. Spatial distribution of frequency in hertz at a time step of 100000 showing the SPP surface wave on the graphene layer.

loss of its SPPs is relatively low; hence, the propagation length could reach dozens of wavelengths of SPPs [17]. For our second example, the infinite graphene sheet is excited by a sinusoidal dipole electric source as the SPP surface source at $30 \mathrm{THz}$ frequency. Our domain has $200 \times 60$ cells and $\Delta x=\Delta y=20 \mathrm{~nm}$. The time step is calculated as $\Delta t=\Delta x /\left(2 c_{0}\right)=3.3 \times 10^{-17}$, and we truncated the domain with eight layer PML cells. In order to avoid spurious reflections from the boundary, the graphene layer is extended to PML regions. Fig. 4 shows the spatial distribution of frequency in hertz at a time step of 100000 when the fields reach steady state. From field distribution, we can easily extract the guided wavelength $\lambda_{\mathrm{SPP}}=29 \times 20 \mathrm{~nm}=580 \mathrm{~nm}$, which is in good agreement with the analytically calculated guided wavelength as $\lambda_{\mathrm{SPP}}=\left(\lambda_{0} / R\left(\left(1-\left(\left(2 / \eta_{0} \sigma_{\mathrm{gr}}\right)\right)^{2}\right)^{1 / 2}\right)\right)=586 \mathrm{~nm}$. $\lambda_{0}$ is the free-space wavenumber [16].

\section{CONCLUSION}

We developed a simple, low-cost formulation based on equivalence between FETD and FDTD to model the graphene, which can be implemented with minimal modifications on an existing FDTD code.

Stability analysis has been performed for the first time unlike the other methods used to model the graphene. Although it is an explicit method, the CFL stability condition has only been changed negligibly.

\section{ACKNOWLEDGMENT}

This work was supported by the Natural Sciences and Engineering Research Council of Canada.

\section{REFERENCES}

[1] K. S. Novoselov, V. I. Fal'ko, L. Colombo, P. R. Gellert, M. G. Schwab, and K. Kim, "A roadmap for graphene," Nature, vol. 490, no. 7419, pp. 192-200, Oct. 2012.

[2] A. Fallahi and J. Perruisseau-Carrier, "Design of tunable biperiodic graphene metasurfaces," Phys. Rev. B, vol. 86, no. 19, p. 195408 , Nov. 2012.

[3] R. Filter, M. Farhat, M. Steglich, R. Alaee, C. Rockstuhl, and F. Lederer, "Tunable graphene antennas for selective enhancement of THz-emission," Opt. Exp., vol. 21, no. 3, pp. 3737-3745, Feb. 2013.

[4] A. Mock, "Padé approximant spectral fit for FDTD simulation of graphene in the near infrared," Opt. Mater. Exp., vol. 2, no. 6, pp. 771-781, 2012.

[5] H. Lin, M. F. Pantoja, L. D. Angulo, J. Alvarez, R. G. Martin, and S. G. Garcia, "FDTD modeling of graphene devices using complex conjugate dispersion material model," IEEE Microw. Wireless Compon. Lett., vol. 22, no. 12, pp. 612-614, Dec. 2012.

[6] G. D. Bouzianas, N. V. Kantartzis, C. S. Antonopoulos, and T. D. Tsiboukis, "Optimal modeling of infinite graphene sheets via a class of generalized FDTD schemes," IEEE Trans. Magn., vol. 48, no. 2, pp. 379-382, Feb. 2012.

[7] V. Nayyeri, M. Soleimani, and O. M. Ramahi, "Modeling graphene in the finite-difference time-domain method using a surface boundary condition," IEEE Trans. Antennas Propag., vol. 61, no. 8, pp. 4176-4182, Aug. 2013.

[8] D. J. Riley and N. W. Riley, "First order models for thin-material sheets and coatings in the finite-element time-domain method," in IEEE AP-S Int. Symp. Dig., vol. 4. Jun. 2004, pp. 3489-3492.

[9] G. W. Hanson, "Dyadic Green's functions and guided surface waves for a surface conductivity model of graphene," J. Appl. Phys., vol. 103, no. 6, p. 064302, 2008.

[10] V. P. Gusynin, S. G. Sharapov, and J. P. Carbotte, "Magneto-optical conductivity in graphene," J. Phys., Condens. Matter, vol. 19, no. 2, p. 026222 , Jan. 2007.

[11] A. Taflove and S. C. Hagness, Computational Electrodynamics: The Finite-Difference Time-Domain Method, 3rd ed. Boston, MA, USA: Artech House, 2005.

[12] M.-F. Wong, O. Picon, and V. F. Hanna, "A finite element method based on Whitney forms to solve Maxwell equations in the time domain," IEEE Trans. Magn., vol. 31, no. 3, pp. 1618-1621, May 1995.

[13] G. Cohen and P. Monk, "Gauss point mass lumping schemes for Maxwell's equations," Numer. Methods Partial Differ. Equ., vol. 14, no. 1 , pp. 63-88, 1998 .

[14] A. Akbarzadeh-Sharbaf and D. D. Giannacopoulos, "Finite-element time-domain solution of the vector wave equation in doubly dispersive media using Möbius transformation technique," IEEE Trans. Antennas Propag., vol. 61, no. 8, pp. 4158-4166, Aug. 2013.

[15] D. Jiao and J.-M. Jin, "A general approach for the stability analysis of the time-domain finite-element method for electromagnetic simulations," IEEE Trans. Antennas Propag., vol. 50, no. 11, pp. 1624-1632, Nov. 2002.

[16] F. Edelvik, R. Schuhmann, and T. Weiland, "A general stability analysis of FIT/FDTD applied to lossy dielectrics and lumped elements," Int. J. Numer. Model., Electron. Netw., Devices, Fields, vol. 17, no. 4, pp. 407-419, 2004.

[17] B. Wang, X. Zhang, X. Yuan, and J. Teng, "Optical coupling of surface plasmons between graphene sheets," Appl. Phys. Lett., vol. 100, no. 13, p. $131111,2012$. 